\title{
DEVELOPMENT OF THE THIRD HARMONIC SC CAVITY AT FERMILAB
}

\author{
N. Solyak, I.Gonin, H. Edwards, M.Foley, T.Khabiboulline, D.Mitchell, J.Reid, L.Simmons, \\ Fermilab, P.O.Box500, Batavia, IL 60510, USA.
}

\section{Abstract}

The third harmonic $3.9 \mathrm{GHz}$ superconducting cavity was recently proposed by DESY for a new generation of high brightness photo-injector (TTF photoinjector-2) to compensate nonlinear distortion of the longitudinal phase space due to RF curvature of the $1.3 \mathrm{GHz}$ TESLA cavities $[1,2]$. Installation of the $3^{\text {rd }}$ harmonic cavity will allow us to generate ultra-short $(<50 \mu \mathrm{m} \mathrm{rms})$ highly charged electron bunches with an extremely small transverse normalized emittance $(<1 \mu \mathrm{m})$. This is required to support a new generation of linear colliders, free electron lasers and synchrotron radiation sources. In this paper we present the current status of the $3^{\text {rd }}$ harmonic cavity being developed at Fermilab. We discuss the design procedure, the building and testing of the copper and niobium halfcells and components, the design of input and HOM couplers.

\section{INTRODUCTION}

The design philosophy of producing highly charged bunches is based on using a long laser pulse to pull a long bunch from the photocathode and thereby reducing the deleterious space charge effect. However, in the accelerating section, the sinusoidal accelerating field profile distorts long bunches. The purpose of the $3^{\text {rd }}$ harmonic de-accelerating section is to compensate for this distortion. In this manner, the injector should generate nC-bunches at an emittance that is four times lower than what existing injectors typically produce.

Fermilab, as part of the TTF collaboration, participates in developing and testing $3^{\text {rd }}$ harmonic cavities at the existing FNPL photoinjector [3]. A number of USA institutions have expressed interest in specific facets of the photoinjector program and intend to form a collaboration to build and operate a $100+\mathrm{MeV}$ highbrightness electron photoinjector at Fermilab.

\section{CAVITY DESIGN}

\section{Design parameters}

The first design of the $3.9 \mathrm{GHz}$ cavity was proposed by DESY[4]. The cavity consists of nine cells with an elliptical cup shape with a $30 \mathrm{~mm}$ diameter iris and a $40 \mathrm{~mm}$ diameter beam pipe. However, Fermilab calculations done for this design show that the coaxial input coupler has to be mounted very close to the end cell to provide the necessary coupling. In an improved design, the iris diameter of the end-cell was increased to $40 \mathrm{~mm}$ with increased distance from the end-cell to the input coupler (Table 1). This leaves more space for welding and mounting of the helium vessel flange. The field distribution in the half-cavity is shown in Fig.1. The design parameters of the $3^{\text {rd }}$ harmonic section are presented in Table 2.

Table 1: Geometry of the cups.

\begin{tabular}{|l|c|c|}
\hline Dimensions in mm & mid-cell & end-cell \\
\hline Iris radius, $a$ & 15.0 & 20.0 \\
\hline Equator radius, $b$ & 35.787 & 35.787 \\
\hline Half-cell length, $h$ & 19.2167 & 19.2167 \\
\hline Curvature at: & \multicolumn{3}{l|}{} \\
\hline Equator-horz/vert semi-axis & $13.6 / 15.0$ & $14.4 / 15.0$ \\
\hline Iris - horz/vert. semi-axis & $4.5 / 6.0$ & $4.5 / 6.0$ \\
\hline
\end{tabular}

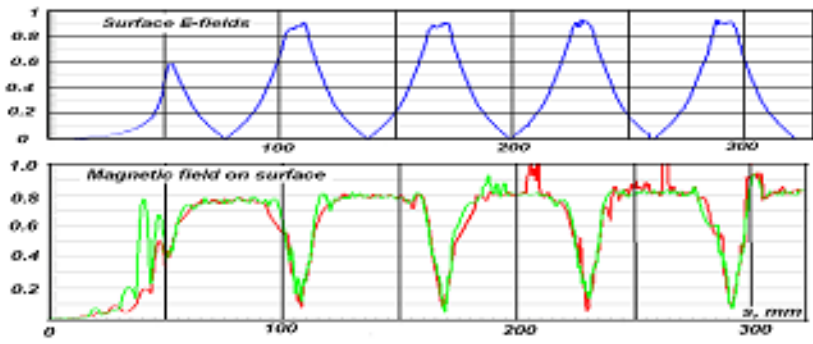

Fig.1. E-field (from the top: in cavity, on axis and on surface) and $\mathrm{H}$-field on surface (bottom). HFSS.

Table 2: $3.9 \mathrm{GHz}$ section parameters.

\begin{tabular}{|l|c|c|}
\hline Number of cavities & & 4 \\
\hline Active Length & $\mathrm{m}$ & 0.346 \\
\hline Gradient & $\mathrm{MV} / \mathrm{m}$ & 20 \\
\hline Phase & degree & -179 \\
\hline R/Q & Ohm & 375 \\
\hline$Q_{\text {ext }}$ for accelerating mode & & $9.5 \mathrm{e}+5$ \\
\hline BBU limit for HOM, Q & & $<1 . \mathrm{e}+5$ \\
\hline Total energy & $\mathrm{MeV}$ & 20 \\
\hline Beam current & $\mathrm{mA}$ & 9 \\
\hline RF power/per cavity & $\mathrm{kW}$ & 11.5 \\
\hline
\end{tabular}

\section{Lorentz forces}

Lorentz forces, caused by electromagnetic fields, may change frequency during the RF pulse. An ANSYS analysis, made for niobium half-cells with fixed boundaries, shows frequency shift of $90 \mathrm{~Hz}$ for a $2.8-\mathrm{mm}$ thickness and $\sim 200 \mathrm{~Hz}$ for a $1.5 \mathrm{~mm}$ thickness. (Fig.2). Electric and magnetic surface fields were taken from HFSS calculations. If free boundaries are assumed, then the frequency shift will be one order of magnitude higher. However, comparison calculations with the experimental results for TESLA and CKM cavities show, that the fixed 
boundaries assumption is more realistic. These calculations show that the rigidity of the cavity, fabricated from $2.8 \mathrm{~mm}$ thick niobium is high enough, even without stiffening rings.

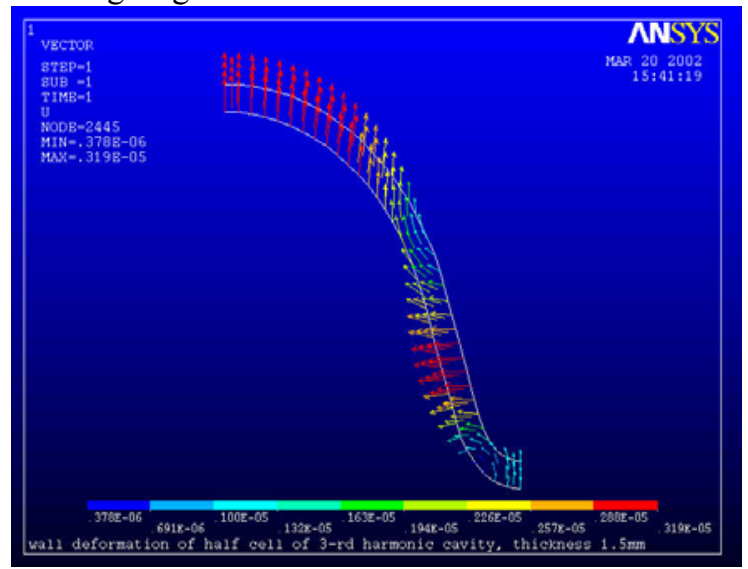

Fig.2. Distribution of surface displacement.

\section{FABRICATION OF COMPONENTS}

\section{Forming of half-cells}

For cup production two different processes are under development. For the conventional deep drawing process, we built a set of dies, machined from 7075-T6 aluminum alloy and formed the cups, using 2.8-mm-thick niobium (RRR 300+) or copper blanks. (Fig.3). To get the required curvature at the iris, the nose of the cup is then coined [5]. An alternative process is hydro-forming of the cups. Advanced Energy Systems, Inc. is developing this process for the $3^{\text {rd }}$ harmonic and CKM cavities.

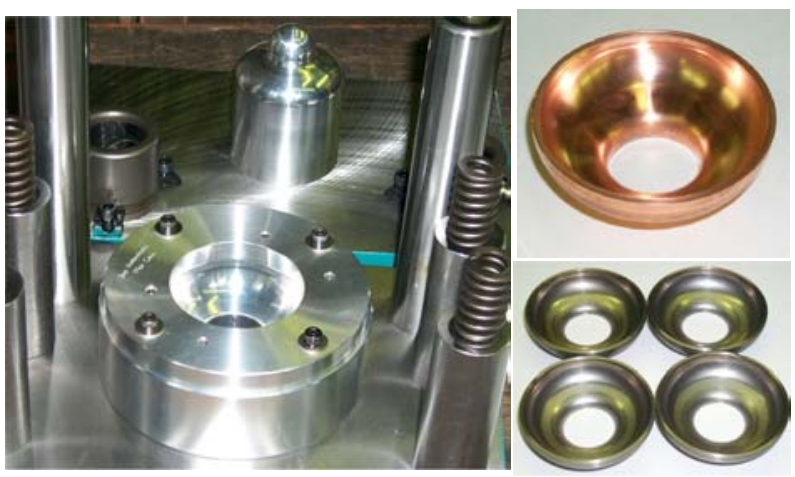

Fig.3. Tooling. Copper and niobium half-cells.

Using the deep drawing technology, many copper and niobium cells have been produced. The shape was carefully monitored on a CMM machine. First results show large profile errors of $\sim 200 \mu \mathrm{m}$, predominately near the iris, where the radius of curvature is only $4.5 \mathrm{~mm}$. This deviation causes a frequency error of $\sim 15 \mathrm{MHz}$. The best and repeatable results were achieved by intermediate annealing followed by re-coining and re-stamping (Fig4.). All 22 copper and 6 niobium cups, produced with this improved technology, have $<1 \mathrm{MHz}$ dispersion in frequency. The actual frequency is well correlated with the length of the cell.

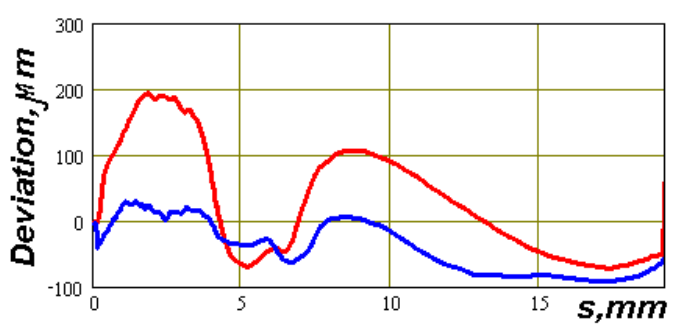

Fig.4. Deviation from design shape after drawing+coining (red), and following annealing+coining + drawing (blue).

\section{Welding of Dumbbells}

Twelve niobium cups were formed to help develop the forming and welding technology. Electron-beam welding parameters were optimized for iris and equator welds (Fig.5). The measured weld shrinkage was $\sim 0.3 \mathrm{~mm} /$ joint at the iris and $\sim 0.2 \mathrm{~mm} /$ joint at the equator with deviation of $\pm 10 \%$. Annealed half-cells had higher shrinkage, but more tests are needed to finalize EBW characteristics.
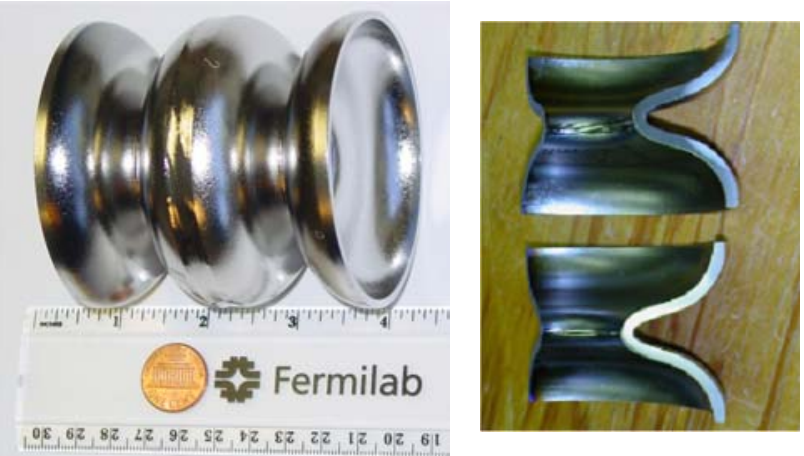

Fig.5. Welded dumbbells

\section{Cavity tuning}

After fabrication, the cavity has to be tuned to obtain the design frequency and field flatness. Limitations in the space between cup pairs, makes it difficult to use a conventional tuning system. A more adequate solution is obtained by squeezing the equator diameter instead of changing the cell length [6]. This type of tuner was designed, built and tested (Fig.6). The tuning clamp consists of 8 curved steel sectors, sliding on a chain. The chain length can be adjusted by a screw. Since the inner surface of this clamp conforms to the cell shape, longitudinal lengthening of the cell is minimal. During the test, a niobium cell was easily tuned up to $35 \mathrm{MHz}$, which is a higher than our goal.

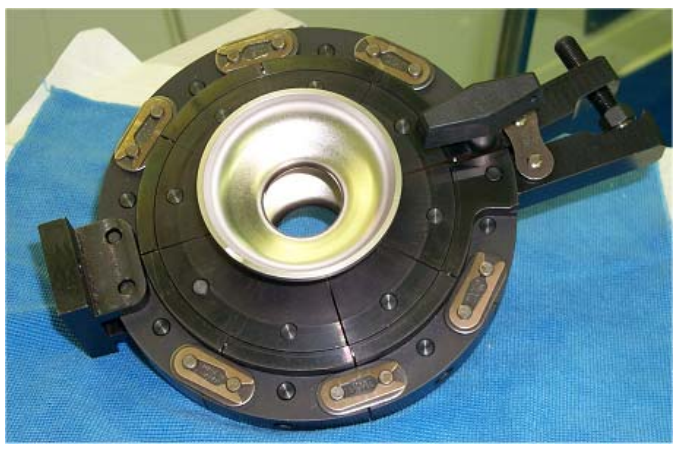

Fig.6 Frequency tuning device. 


\section{Copper model}

Building a copper model is the first step in developing fabrication, tooling, RF control and tuning. Copper and niobium have similar mechanical properties, but copper is cheaper and has a higher conductivity at room temperature. A general view of the copper model and some components are shown in figure 7.

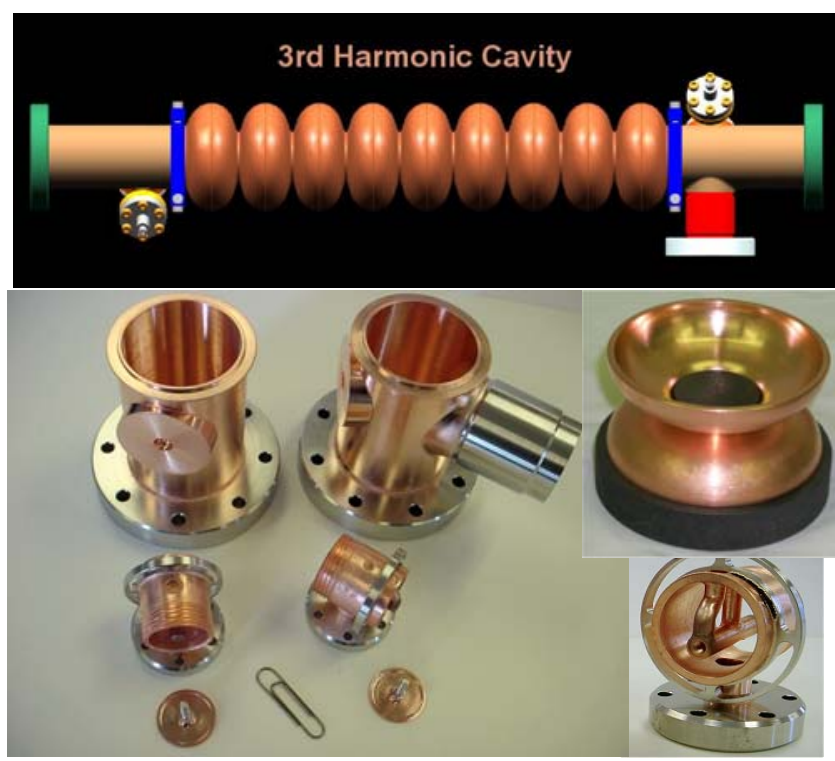

Fig.7. Cavity, end-tubes, dumbbells and HOM couplers.

End-tubes are decoupled from the cavity body to give us the ability to rotate or replace components if needed. HOM couplers are also rotatable. Measured RF properties of the coupler are in a good agreement with the calculations. All components are brazed, tested and ready for final brazing.

\section{Input coupler}

Fig. 8 shows the design of the coaxial, adjustable input coupler, we are developing for $3.9 \mathrm{GHz}$ cavity. This is $50 \Omega$ coaxial line with a $30 \mathrm{~mm}$ outer diameter to prevent the excitation of asymmetrical modes. For the cold window, we adopted the cylindrical ceramics of TESLA's coupler. For the warm window we are planning to use a standard waveguide window designed by CPI for a 3.9 GHz klystron.

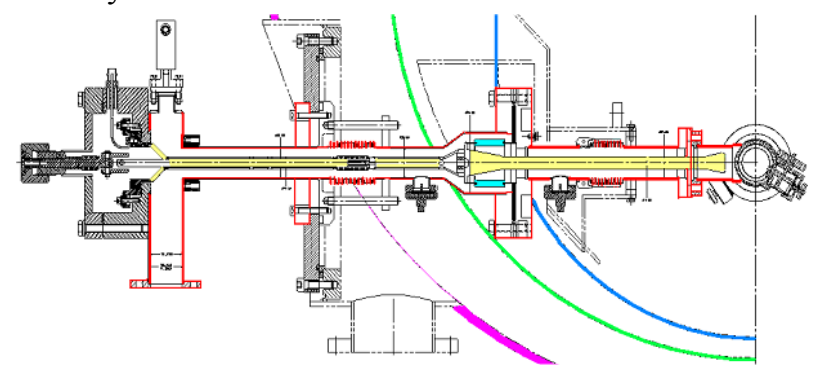

Fig. 8. Coaxial input coupler.

The coupler has pick-ups and light detection and is designed with DC biasing to suppress multipactor (MP). A lower power level should facilitate MP problems, but we need more detailed calculations to prove this statement. Bellows, in the assembly, allow us to move the antenna back and forth $( \pm 2.5 \mathrm{~mm})$ and adjust Qext by a factor of five. The current design is quite complicated and after a discussion with W.D. Moller, we are working on a simple non-adjustable version of this coupler.

\section{HOM analysis}

Trapped High Order Modes (HOM) may excite BBU instability in the accelerator. Beam dynamics analysis gives us an upper limit of Qext (Table.2). Using HFSS, we have analyzed high order modes in 2-D model (R/Q calculations) and 3-D (R/Q and $\left.\mathrm{Q}_{\text {external }}\right)$ up to $9 \mathrm{GHz}$ [7,8]. 3-D geometry includes the input coupler and two HOM couplers with mirrored symmetrical antennas. The cavity in this model is excited by the beam with $2 \mathrm{~mm}$ vertical or horizontal off-sets. Analysis shows that if we apply electric or magnetic boundaries at the end of cavity, then few modes in the third passband have Q ext above the BBU limit. For a more realistic assumption, "open" boundaries, all dipole modes in three first pass-band will be significantly damped with the maximal $\mathrm{Q}_{\mathrm{ext}}=2400$ (figure 9).

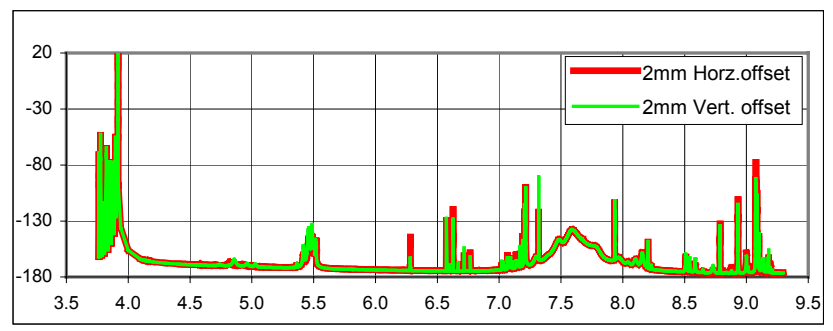

Fig.9. Field amplitude in cavity (dB) vs. frequency $(\mathrm{GHz})$.

\section{CONCLUSION}

Fermilab is making good progress in developing a superconducting $3.9 \mathrm{GHz}$ accelerating cavity [9].

\section{REFERENCES}

[1] M.Ferrario et al., "Conceptual Design of the XFEL Photoinjector", TESLA-FEL-01-03.

[2] F.Floettmann et al.,"Generation ultrashort electron bunches by cancellation of nonlinear distortions in the longitudinal phase space", TESLA-FEL-01-06.

[3] J-P.Carniero et al., "First results of the Fermilab high brightness RF photoinjector", Proc. PAC'99, p.2027.

[4]J.Sekutowicz et. al.,"A design of a $3^{\text {rd }}$ harmonic cavity for TTF2 Photoinjector". TESLA-FEL, 2002-05.

[5] H. Padamsee et al., "RF superconductivity for accelerators", 1998.

[6] H.Kaiser, "New approaches to tuning of TESLA resonators", Proc. 9th Workshop on RFSC, Santa Fe, Nov.1999.

[7] T. Khabibouline et.al., "Higher Order Modes of a $3^{\text {rd }}$ harmonic Cavity with an increased end-cup iris", preprint FNAL,2003.

[8]W.F.O.Muller et.al., This conference, RPPB071.

[9] http://www-td.fnal.gov/lc/sc/lc.html 